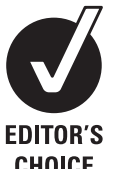

CHOICE

\begin{abstract}
${ }^{1}$ Agència de Salut Pública de Barcelona, Barcelona, Spain

${ }^{2}$ Programa de Doctorado en Biomedicina, Universitat

Pompeu Fabra, Barcelona, Spain ${ }^{3}$ CIBER Epidemiología y Salud Pública (CIBERESP), Barcelona, Spain

${ }^{4}$ Ministerio de Sanidad y Consumo, Madrid, Spain ${ }^{5}$ Centro de Salud Pública de Alzira, Alzira, Spain ${ }^{6}$ Instituto de Diagnóstico Ambiental y Estudios del Agua, CSIC, Barcelona, Spain ${ }^{7}$ Dirección General de Tráfico, Madrid, Spain

${ }^{8}$ Universitat Pompeu Fabra, Barcelona, Spain
\end{abstract}

\section{Correspondence to}

Dr Katherine Pérez, PI Lesseps,

1; 08023 Barcelona, Spain;

cperez@aspb.cat

This paper will be included in the thesis of one of the authors (AMN) performed at the Pompeu Fabra University (UPF).

Accepted 19 November 2009 Published Online First 3 December 2009

\title{
Road safety in the political agenda: the impact on road traffic injuries
}

\author{
Ana M Novoa, ${ }^{1,2}$ Katherine Pérez, ${ }^{1,3}$ Elena Santamariña-Rubio, ${ }^{1}$ \\ Marc Marí-Dell'Olmo, ${ }^{1,3}$ Rogelio Cozar, ${ }^{4}$ Josep Ferrando, ${ }^{3}$ Rosana Peiró, ${ }^{3,5}$ \\ Aurelio Tobías, ${ }^{6}$ Pilar Zori, ${ }^{7}$ Carme Borrell ${ }^{1,3,8}$
}

\begin{abstract}
Background This paper aims at assessing the effectiveness of the package of road safety measures implemented after road safety was included in the political agenda in the year 2004 on the number of road traffic-injured people in Spain.

Methods An evaluation study was performed using an interrupted time-series design. The study population was people injured in road traffic crashes in Spain between 1 January 2000 and 31 December 2006. The road traffic crashes database of the General Directorate for Traffic was used. The dependent variable was the monthly number of people injured, stratified by sex, age, severity and type of road user. The explanatory variable (intervention) compared the post-intervention period (2004-6) with the pre-intervention period (2000-3). Quasi-Poisson regression models were adjusted, controlling for time trend and for seasonality.
\end{abstract}

Results Results show a reduction in the risk of being injured for both men (RR 0.91; 95\% $\mathrm{Cl} 0.87$ to 0.95) and women (RR 0.89; $95 \% \mathrm{Cl} 0.85$ to 0.94). Risk reductions were observed across all age groups and all road users, except for pedestrians.

Conclusions The present study suggests that prioritising road safety reduces the number of people injured in road traffic collisions.

Road traffic injuries cause great mortality and morbidity worldwide. ${ }^{1} 2$ In Spain, they are the primary cause of death among individuals aged $1-39$ years, the third for individuals aged $40-59$ years and the fifth for individuals aged 60-69 years. ${ }^{3}$ In addition, they are the primary cause of potential years of life lost in men, the second in women. ${ }^{4} 5$

To improve road safety, the White Paper on European transport policy established the target of reducing road fatalities by $50 \%$ by the year 2010 (compared with 2001). ${ }^{6}$ The Road Safety Action Programme describes specific measures-related to road user behaviour (mainly based on police enforcement), vehicle safety and road infrastructure-aimed at achieving this target. ${ }^{7}$ A strong and sustained political will is required to achieve these targets and ensure that road safety is given enough priority, including appropriate funding, necessary legislative changes and a capable bureaucracy. ${ }^{8-10}$

Following the approval of the Road Safety Action Programme, the Spanish government established road safety as a political priority, and created the Road Safety Special Measures $2004-5^{11}$ and the Road Safety Strategic Programme 2005-8, ${ }^{12}$ the main goal of which is to achieve a $40 \%$ reduction in road fatalities by the year 2008 (compared with 2003), and lists 182 actions - to be progressively implemented-included in 10 strategic areas: (1) road safety education; (2) road safety awarenes; (3) surveillance and control; (4) vehicle safety; (5) road infrastructures and improvement in road safety information and management; (6) road safety in the field of transport and labour; (7) attention to people injured in accidents and their families; (8) road safety research and analysis; (9) participation of society and (10) coordination between administrations.

Before the year 2004 the implemented interventions were mostly based on road safety normative, such as the establishment of illegal blood alcohol concentration levels, speed limits, or making it compulsory to use passive safety devices. Also, road infrastructure and healthcare delivery were improved. ${ }^{13}$ However, road safety enforcement measures were mostly implemented from the year 2004 on (eg, the number of new speed cameras installed increased from four during the year 2004 up to 197 during 2006; the number of alcohol checkpoints performed over the number of registered drivers increased from $11.1 \%$ in 2003 to $15.8 \%$ in 2006). ${ }^{14} 15$ Further road safety normative was also approved: in 2004 standardised child safety seats and bicycle helmets on non-urban roads were made compulsory, and in 2006 the penalty points system was implemented and the life period for school buses was set at a maximum of 10 years. ${ }^{16}$

Although other countries have undertaken similar initiatives to those implemented in Spain, to date the overall effect of the actions implemented following road safety prioritisation has not yet been assessed.

The objective of this paper is to assess the impact of road safety prioritisation in the year 2004 on the number of traffic-injured people in Spain. In particular, the effectiveness of the package of road safety interventions implemented after the introduction of road safety in the political agenda will be assessed. Differences in the effectiveness with respect to gender, age, injury severity and the type of road user will also be assessed.

\section{MATERIALS AND METHODS Study design and population}

An evaluation study was performed using an interrupted time-series design. The study population was people injured (fatal and non-fatal) in traffic crashes in Spain between January 2000 and December 2006. 


\section{Sources of information}

The road traffic crashes database of the Dirección General de Tráfico (General Directorate for Traffic) was used, which contains data - collected by police officers-for injury collisions: the characteristics of the collision, the vehicle and the subjects involved.

Vehicle fleet and the number of new vehicle registrations, used as proxies for exposure, were available at the Dirección General de Tráfico home page. ${ }^{17}$

\section{Variables}

The dependent variable was the number of people injured in traffic collisions. This variable was stratified according to sex, age (0-13 years, $14-15$ years (allowed to ride mopeds), $16-17$ years (also allowed to ride motorcycles $\leq 125 \mathrm{cc}$ ), 18-29 years (allowed to drive any type of vehicle), 30-44 years, 45-64 years, 65-74 years, >74 years), type of road user (car user, motorcycle user, moped user, pedestrian) and severity (slight, serious, fatal-in $24 \mathrm{~h}$ ). The police classify as seriously injured those who are hospitalised more than $24 \mathrm{~h}$.

The explanatory variable was the intervention, which in this case includes overall interventions implemented after road safety was included in the political agenda (compared with those implemented before it). A dummy variable was created to compare the post-intervention period (January 2004-December 2006) with the pre-intervention period (January 2000-December 2003).

An exhaustive database containing all of the implemented road safety measures in Spain is not available. Moreover, although the national government is in charge of road safety normative, the specific road safety actions performed also depend on the local governments and vary greatly depending on the region. Consequently, given that it is not possible to define clearly the interventions implemented before and after road safety prioritisation, these two periods have to be thought of as two black boxes that mainly differ in that the intensity of road safety enforcement was much greater in the post-intervention period.

Several socioeconomic variables were accounted for as potential confounding factors: gasoline and gas-oil consumption, unemployment rate and the gross national product.

Since July 2005, there is a new protocol for police data collection aimed at improving the reporting of traffic crashes in Spain. This has probably involved an increase in the number of collisions registered, mostly involving slight injuries, but also serious injuries. To account for the effect of this protocol, certain analyses were repeated including a dummy variable in the model that compared the period before (January 2000-June 2005) and after (July 2005-December 2006) its approval.

\section{Statistical analysis}

Monthly time-series analyses were carried out using Poisson regression models adjusted for over-dispersion (quasi-Poisson). ${ }^{18}$

The absolute number of people injured was compared throughout the time series. Potential confounding by time trend and seasonal patterns was controlled for using a linear trend and sine and cosine functions. ${ }^{19}$ The model can thus be summarised as follows:
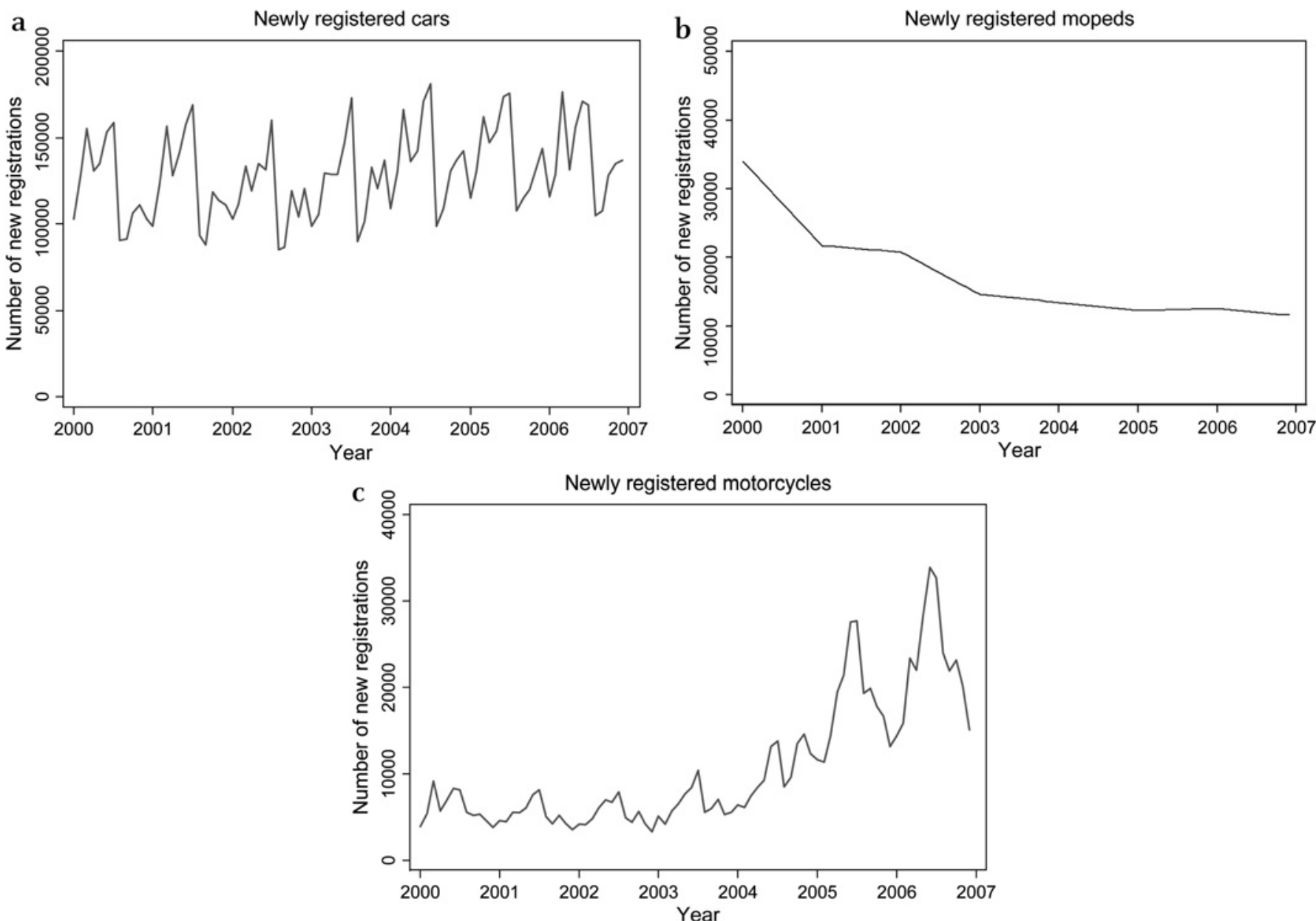

Figure 1 Monthly number of new car, motorcycle and moped registrations. Spain 2000-6. 
Table 1 Distribution of road traffic-injured people by age, injury severity and type of road user. Spain 2000-6

\begin{tabular}{|c|c|c|c|c|}
\hline & \multicolumn{4}{|l|}{ Police data* } \\
\hline & \multicolumn{2}{|l|}{ Men $(n=665788)$} & \multicolumn{2}{|c|}{ Women $(n=345312)$} \\
\hline & Monthly median & $\%$ & Monthly median & $\%$ \\
\hline \multicolumn{5}{|l|}{ Age (years) } \\
\hline $0-13$ & 244 & 3.3 & 193 & 5.1 \\
\hline $14-15$ & 134 & 1.8 & 73 & 2.0 \\
\hline $16-17$ & 379 & 5.2 & 148 & 3.9 \\
\hline $18-29$ & 3039 & 39.8 & 1408 & 36.1 \\
\hline $30-44$ & 2082 & 27.4 & 981 & 25.0 \\
\hline $45-64$ & 1209 & 15.9 & 717 & 18.2 \\
\hline $65-74$ & 320 & 4.2 & 228 & 5.8 \\
\hline$\geq 75$ & 180 & 2.4 & 158 & 4.0 \\
\hline \multicolumn{5}{|c|}{ Injury severity according to police criteria } \\
\hline Slight & 6146 & 77.9 & 3444 & 84.0 \\
\hline Serious & 1472 & 18.6 & 562 & 14.0 \\
\hline Fatal (24 h) & 280 & 3.5 & 78 & 2.0 \\
\hline \multicolumn{5}{|l|}{ Type of road user } \\
\hline Car user & 4139 & 54.2 & 2741 & 67.3 \\
\hline Motorcycle user & 781 & 10.8 & 139 & 3.6 \\
\hline Moped user & 1397 & 18.7 & 461 & 11.5 \\
\hline Pedestrian & 507 & 6.5 & 474 & 11.6 \\
\hline Others & 968 & 9.8 & 291 & 6.0 \\
\hline
\end{tabular}

*35 800 (3.4\%) Subjects were not identified as being male or female.

$$
\begin{gathered}
\ln \left[E\left(Y_{t}\right)\right]=\beta_{0}+\beta_{1} t+\sum_{k=1}^{6}\left[\beta_{2 k} \sin \left(\frac{2 k \pi t}{T}\right)+\right. \\
\left.\beta_{3 k} \cos \left(\frac{2 k \pi t}{T}\right)\right]+\beta_{4} X_{t}+\beta_{5} X_{t} t+\sum_{j}\left(\beta_{6 j} Z_{j t}\right)+\epsilon_{t}
\end{gathered}
$$

where $t$ is the time period ( $t=1$ for the first month of the series, $t=2$ for the second month, etc), $k$ takes values between 1 and 6 (eg, $k=1$ for annual seasonality; $k=2$ for 6 -monthly seasonality), $\mathrm{T}$ is the number of periods described by each sinusoidal function (eg, $T=12$ months), $X_{t}$ identifies the pre and post-intervention periods $\left(X_{t}=1\right.$ for the post-intervention period), $Z_{j t}$ other covariables introduced (socioeconomic variables, new protocol for police data collection), $j$ the number of co-variables introduced, and $\epsilon$ the error term. Only statistically significant terms were included in the final model.

RR and their 95\% CI were derived from the adjusted models. Two different RR were obtained, one corresponding to the mean change $\left(\beta_{4}+\beta_{5} t_{0}\right.$, where $t_{0}$ identifies the month in which the intervention took place), which indicates the change in the mean number of people injured during the first month of the post-intervention period compared with the previous month (adjusting for time trend and seasonality; short-term effect), and another corresponding to trend change $\left(\beta_{5}\right)$, which indicates the change in the time trend between the two periods (long-term effect). ${ }^{20}$ The RR for the mean change should not be confused with the mean change in the overall number of people injured throughout the whole post-intervention period.

The number of people injured prevented by road safety prioritisation was calculated as the difference between the observed and expected numbers of people injured throughout the whole post-intervention period-thus summarising the short and long-term effect. The expected numbers were predicted with the statistical models.

Statistical analyses were carried out using Stata statistical software, release $10 .^{21}$

\section{Exposure-adjusted analyses}

The analysis of the absolute number of people injured assumes that exposure has remained stable throughout the study period. As this assumption might be unrealistic, analyses were also performed using as denominators the monthly car, motorcycle and moped fleet and the monthly number of new car, motorcycle and moped registrations. However, appropriate exposure denominators (ie, kilometres travelled by vehicle) were not available (information is only available for non-urban roads). These denominators where included in the models as an offset.

Given that the moped fleet was not available for the whole study period and that similar results were obtained using vehicle fleet and vehicle registrations, only registrations-adjusted results will be shown (figure 1).

\section{RESULTS}

During the study period 1046900 people were injured in traffic collisions (annual median of 152264 ), 66.8\% of them being men and $65.1 \%$ between 18 and 44 years of age (table 1 ).

The type of road user varied with the age and sex of the person injured (figure 2): individuals were mostly car users (54.2\% men, $67.1 \%$ women), with the exception of those aged $14-15$ and $16-17$ years, who were mostly moped users $(66.0 \%$ and $41.2 \%$ in boys and girls from 14 to 15 years, respectively; $76.8 \%$ and $50.7 \%$ from 16 to 17 years). Also, the proportion of pedestrians was higher among individuals aged from 0 to

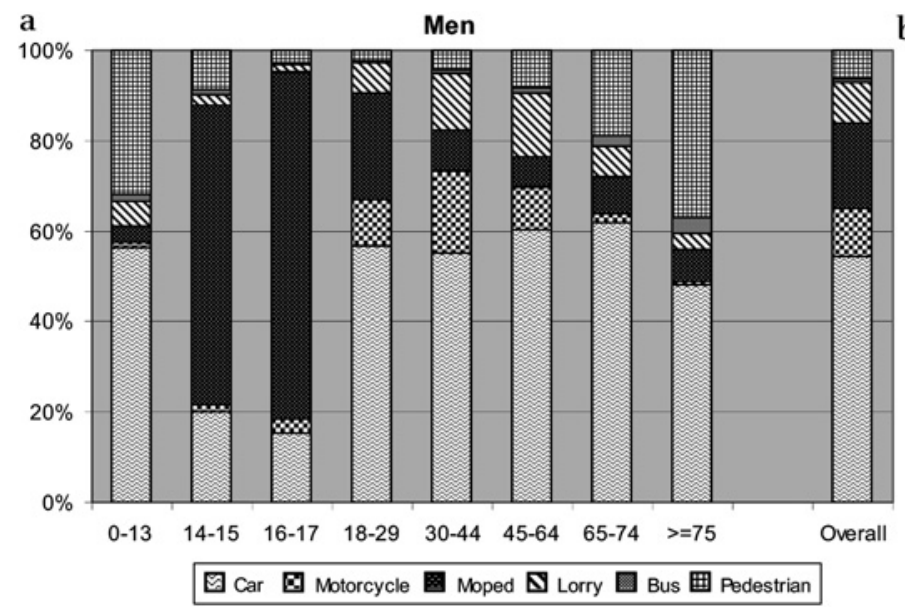

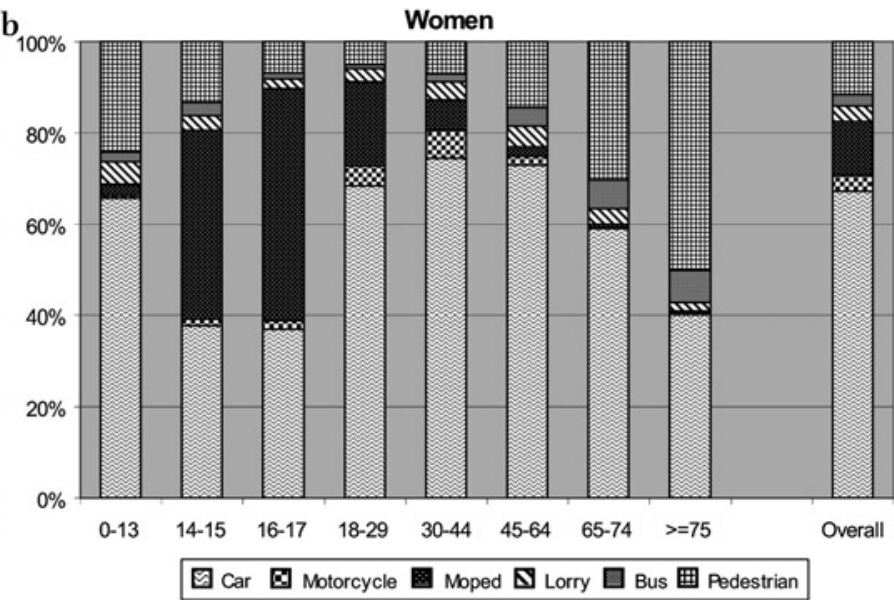

Figure 2 Distribution of type of road user involved in road traffic crashes in Spain by age and gender. Spain 2000-6. 
Table 2 Adjusted RR for people being injured in traffic collisions in the post-intervention period compared with the pre-intervention period, regarding mean change and time trend change $\dagger$ between the pre and post-intervention period, according to injury severity, age and type of road user. Spain $2000-6$

\begin{tabular}{|c|c|c|c|c|c|c|c|c|c|c|c|c|}
\hline & \multicolumn{6}{|l|}{ Men } & \multicolumn{6}{|l|}{ Women } \\
\hline & \multicolumn{2}{|l|}{ Mean change } & \multicolumn{4}{|c|}{ Trend change } & \multicolumn{2}{|l|}{ Mean change } & \multicolumn{4}{|c|}{ Trend change } \\
\hline & \multirow[b]{2}{*}{ RR $(95 \%$ CI) } & \multirow[b]{2}{*}{ p Value } & \multirow[b]{2}{*}{ Pre $(\%)$} & \multirow[b]{2}{*}{ Post (\%) } & \multicolumn{2}{|c|}{ Relative change } & \multirow[b]{2}{*}{ RR $(95 \% \mathrm{Cl})$} & \multirow[b]{2}{*}{ p Value } & \multirow[b]{2}{*}{ Pre (\%) } & \multirow[b]{2}{*}{ Post (\%) } & \multicolumn{2}{|c|}{ Relative change } \\
\hline & & & & & $\%$ & p Value & & & & & $\%$ & p Value \\
\hline Overall & $0.91(0.87$ to 0.95$)$ & $<0.001$ & -0.08 & $0.19 *$ & 0.27 & 0.004 & 0.89 (0.85 to 0.94$)$ & $<0.001$ & $0.17^{*}$ & $0.24 *$ & 0.07 & 0.514 \\
\hline \multicolumn{13}{|l|}{ Injury severity } \\
\hline Slight & $0.92(0.87$ to 0.96$)$ & 0.001 & -0.05 & $0.27^{*}$ & 0.32 & 0.002 & $0.90(0.86$ to 0.95$)$ & $<0.001$ & $0.21^{*}$ & $0.34^{*}$ & 0.13 & 0.288 \\
\hline Serious & 0.88 (0.83 to 0.92$)$ & $<0.001$ & $-0.15^{*}$ & 0.00 & 0.15 & 0.198 & 0.82 (0.76 to 0.88$)$ & $<0.001$ & -0.02 & -0.20 & -0.18 & 0.252 \\
\hline Fatal (24 h) & 0.95 (0.88 to 1.01$)$ & 0.120 & $-0.16^{*}$ & $-0.56^{*}$ & -0.40 & 0.009 & $0.99(0.87$ to 1.12$)$ & 0.811 & -0.21 & $-1.17^{*}$ & -0.96 & 0.001 \\
\hline \multicolumn{13}{|l|}{ Type of vehicle } \\
\hline Car & 0.88 (0.83 to 0.93$)$ & $<0.001$ & $0.18^{*}$ & -0.19 & -0.37 & 0.005 & $0.84(0.79$ to 0.90$)$ & $<0.001$ & $0.36^{*}$ & 0.13 & -0.23 & 0.106 \\
\hline Motorcycle & 0.95 (0.89 to 1.02$)$ & 0.146 & $-0.21^{*}$ & $1.76^{*}$ & 1.97 & $<0.001$ & $0.90(0.81$ to 0.99$)$ & 0.035 & -0.14 & $1.98^{*}$ & 2.12 & $<0.001$ \\
\hline Moped & $0.99(0.93$ to 1.04$)$ & 0.607 & $-0.80^{*}$ & -0.12 & 0.68 & $<0.001$ & $1.02(0.95$ to 1.09$)$ & 0.628 & $-0.55^{*}$ & -0.09 & 0.46 & 0.001 \\
\hline Pedestrian & $0.97(0.91$ to 1.04$)$ & 0.407 & $-0.23^{*}$ & -0.06 & 0.17 & 0.241 & $0.98(0.92$ to 1.04$)$ & 0.481 & $-0.19^{*}$ & 0.21 & 0.40 & 0.004 \\
\hline
\end{tabular}

Post, post-intervention period; Pre, pre-intervention period. Pre-intervention period: 1 January 2000-31 December 2003; post-intervention period: 1 January $2004-31$ December 2006. ${ }^{*} \mathrm{p}<0.05$.

†Time trends in the pre (Pre(\%)) and post (Post(\%)) intervention periods are expressed as the mean percentage increase/decrease in the number of people injured per month, statistically significant trends being indicated with an asterisk $\left({ }^{*}\right)$. Relative change is expressed as the mean percentage increase/decrease in the number of people injured per month in the post-

intervention period compared with that in the pre-intervention period.

13 years $(31.9 \%$ in boys, $24.2 \%$ in girls), decreasing with age, and increasing again from 45 years on, up to $37.3 \%$ in men and $50.2 \%$ in women aged over 74 years. The proportion of injured motorcycle or moped users was higher in men than in women, whereas the proportion of injured car users and pedestrians was higher in women $(p<0.05)$.
Figure 3 Monthly number of observed people injured due to a road traffic collision and time trend $(95 \% \mathrm{Cl})$ in the pre and post-intervention periods, according to injury severity and gender. Spain 2000-6.
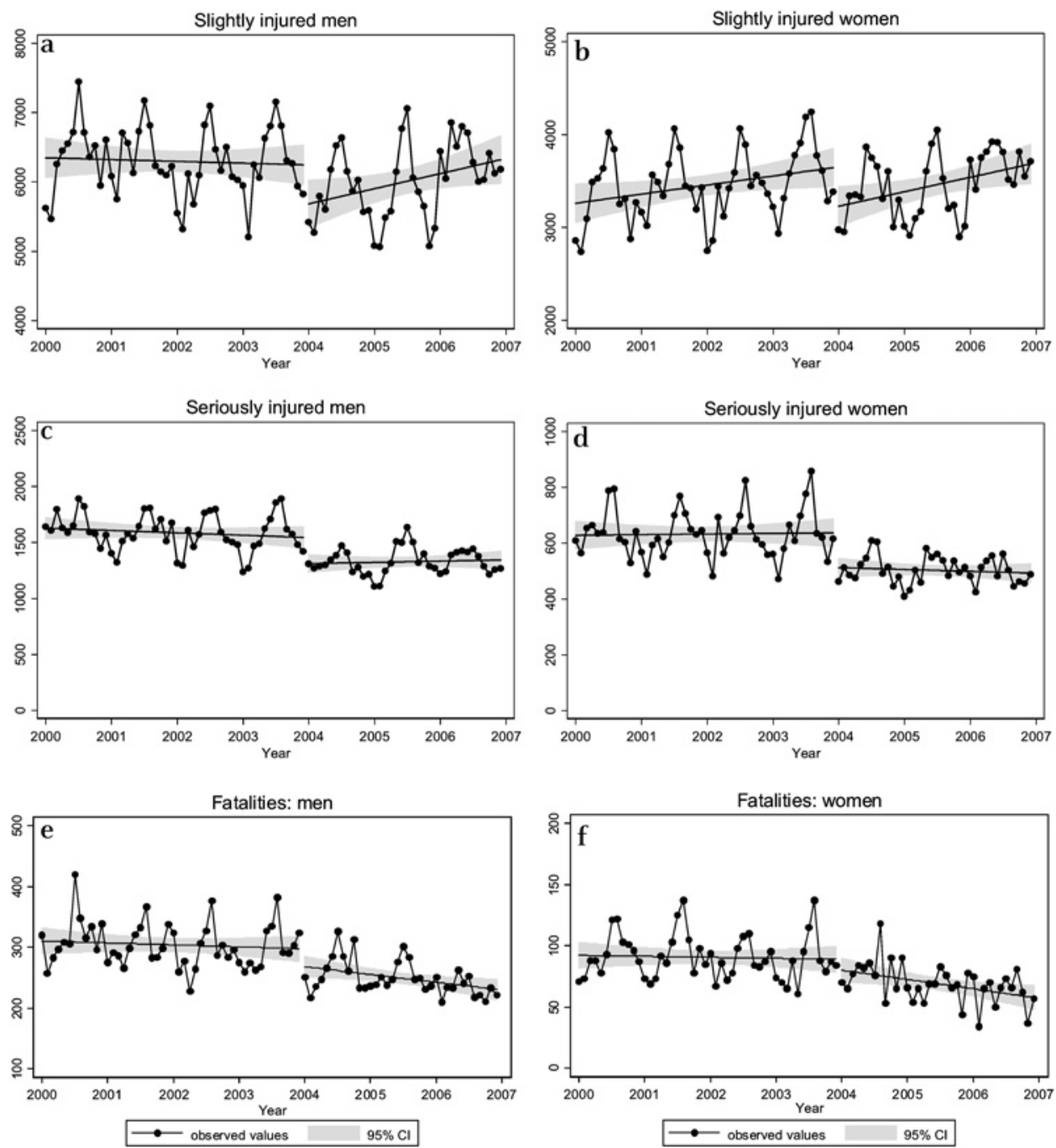
Figure 4 Monthly number of observed people injured due to a road traffic collision and time trend $(95 \% \mathrm{Cl})$ in the pre and post-intervention periods, according to type of road user and gender. Spain 2000-6.
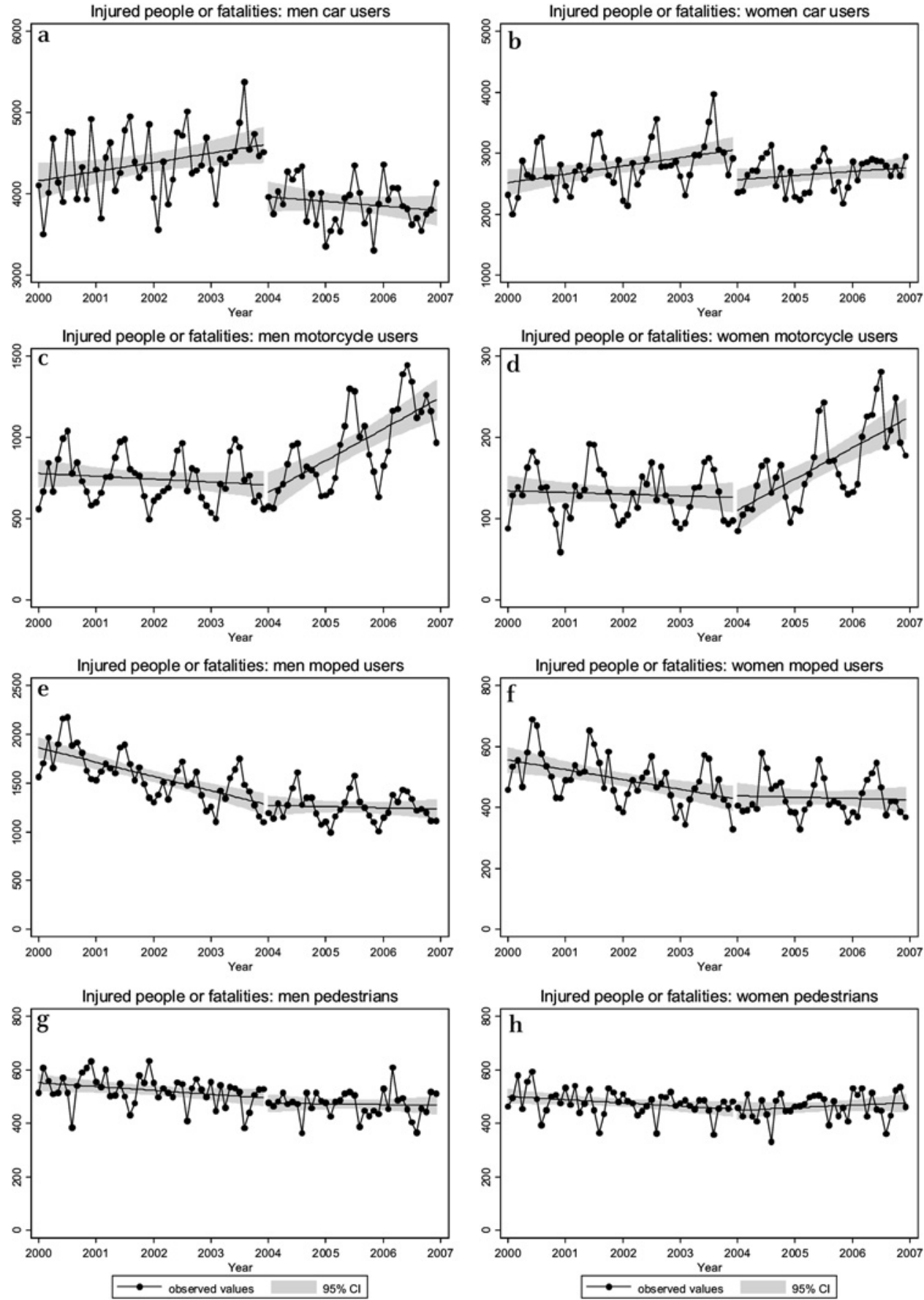

\section{Sex and severity}

In men, a reduction in the risk of being injured was observed during the first month of the post-intervention period (mean change) (RR $0.91 ; 95 \%$ CI 0.87 to 0.95 ). However, a $0.27 \%$ significant increase in the risk of being injured was observed regarding the time trend (table 2 ).

Taking injury severity into account, results among slightly injured men followed a similar pattern compared with overall results. A statistically significant reduction in the risk of being injured in the post-intervention period was observed in the mean number of seriously injured men and in the time trend for male fatalities (table 2 and figure 3).

Results for the type of road user showed a statistically significant reduction in the risk of being injured among male car users in both the mean number and the time trend. Conversely, the time trend for motorcycle and moped users showed a significant risk increase. The number of injured male pedestrians stayed stable throughout the pre and post-intervention periods (table 2 and figure 4).

In women, a reduction in the risk of being injured was observed during the first month of the post-intervention period (RR 0.89; $95 \%$ CI 0.85 to 0.94 ), along with a non-significant $0.07 \%$ increase in the risk of being injured regarding the time trend. Results for injury severity and type of road user were similar to those observed in men (table 2 and figures 3 and 4), although with some differences: the time trend for overall and slightly injured women remained stable throughout the pre and post-intervention periods, and a significant increase was observed in the time trend for women pedestrians injured. On the whole, larger risk reductions were observed among women than among men. 
Table 3 Prevented $\dagger$ numbers of men and women injured in road traffic collisions in the post-intervention period. Spain 2000-6

\begin{tabular}{|c|c|c|c|c|}
\hline & \multicolumn{2}{|l|}{ Men } & \multicolumn{2}{|l|}{ Women } \\
\hline & $\mathbf{N}$ & $\% \neq$ & $\mathbf{N}$ & $\% \neq$ \\
\hline Overall & 9850 & 3.5 & $14779 *$ & 9.3 \\
\hline \multicolumn{5}{|l|}{ Injury severity } \\
\hline Slight & 3311 & 1.5 & $10136^{*}$ & 7.5 \\
\hline Serious & $5414^{*}$ & 10.2 & 4299* & 19.2 \\
\hline Fatal $(24$ h) & $1167^{*}$ & 11.5 & $475^{*}$ & 16.1 \\
\hline \multicolumn{5}{|l|}{ Type of vehicle } \\
\hline Car & $28405^{*}$ & 16.9 & $19230^{*}$ & 16.7 \\
\hline Motorcycle & $-9617^{*}$ & -39.3 & $-1445^{*}$ & -31.8 \\
\hline Moped & $-4847^{*}$ & -12.0 & $-1521^{*}$ & -10.8 \\
\hline Pedestrian & 1 & 0.0 & -921 & -5.9 \\
\hline \multicolumn{5}{|c|}{$\begin{array}{l}{ }^{*} p<0.05 \text {. } \\
\dagger \text { Negative numbers indicate an excess of people injured in the post-intervention period } \\
\text { compared with the expected, according to the numbers observed in the pre-intervention } \\
\text { period. } \\
\neq \text { Calculated as the number of prevented people injured over the expected number of people } \\
\text { injured in the post-intervention period. }\end{array}$} \\
\hline
\end{tabular}

Similar results were obtained when adjusting the approval of the new protocol for police data collection in the models.

\section{Age}

As the distribution of the type of road user varied between age groups (figure 2), the age analyses were stratified by the type of road user. There were no notable differences between age groups in any of the road user categories considered (data not shown).

\section{Prevented number of people injured}

Almost 25000 people injured and more than 1500 deaths were prevented during the post-intervention period attributable to road safety prioritisation, representing a $5.6 \%$ and $12.5 \%$ reduction with respect to the expected numbers, respectively (table 3). Larger reductions were observed among fatalities and seriously injured people compared with slightly injured people and among women compared with men. The number of people injured was greater than expected among motorcycle users, moped users and women pedestrians.

\section{Exposure-adjusted results}

When the number of new vehicle registrations was used as an exposure denominator, in both men and women motorcycle users, significant risk reductions were observed for both the mean number (RR 0.64 in men, RR 0.60 in women) and the time trend $(1.21 \%$ and $1.05 \%$ monthly reductions, respectively). Similar results were observed for moped users (RR 0.68 and RR $0.71 ; 2.68 \%$ and $2.90 \%$ monthly reductions) and for car users ( $R R \quad 0.61$ and $R R \quad 0.60 ; 3.61 \%$ and $3.45 \%$ monthly reductions) (table 4).

\section{DISCUSSION}

The present study suggests that overall road safety interventions implemented following road safety political prioritisation reduced road traffic-injured people in Spain. Effectiveness was shown across all injury severity categories, age groups and road users, except for pedestrians.

Although a large number of studies have assessed the effectiveness of single or combined interventions, to our knowledge, this is the first study to assess the effectiveness of prioritising road safety.

\section{Effectiveness of road safety prioritisation \\ Sex and severity}

Larger risk reductions were observed among women. This could be explained by their higher willingness for behaviour change. ${ }^{22} 23$

The severely injured or killed people showed greater risk reductions than the slightly injured, which could be partly explained because, among the implemented interventions, some aimed at reducing injury severity. These interventions are expected to reduce the proportion of collisions that result in fatal or serious injuries, thereby increasing the number of slightly injured people and, to a lesser extent, the number of seriously injured people. Also, it could be due to the new protocol for police data collection. However, the models adjusted and unadjusted by the new protocol showed limited differences, which could be explained by a lag-time effect with respect to the consequences of the protocol, or because the variable also includes the effect of other events such as the penalty points system.

Type of road user and exposure-adjusted results

Unadjusted results showed risk reductions among car users. However, large risk increases were observed regarding time trend

Table 4 Adjusted RR for people being injured in traffic collisions in the post-intervention period compared with the pre-intervention period, regarding mean change and time trend changet between the pre and post-intervention period, according to type of vehicle. Comparison of the results obtained from the original models with those from models which include the number of new vehicle registrations as an exposure denominator. Spain $2000-6$

\begin{tabular}{|c|c|c|c|c|c|c|c|c|c|c|c|c|}
\hline & \multicolumn{6}{|l|}{ Men } & \multicolumn{6}{|l|}{ Women } \\
\hline & \multicolumn{2}{|l|}{ Mean change } & \multicolumn{4}{|c|}{ Trend change } & \multicolumn{2}{|l|}{ Mean change } & \multicolumn{4}{|c|}{ Trend change } \\
\hline & \multirow[b]{2}{*}{ RR $(95 \%$ Cl) } & \multirow[b]{2}{*}{ p Value } & \multirow[b]{2}{*}{ Pre (\%) } & \multirow[b]{2}{*}{ Post (\%) } & \multicolumn{2}{|c|}{ Relative change } & \multirow[b]{2}{*}{ RR (95\% Cl) } & \multirow[b]{2}{*}{ p Value } & \multirow[b]{2}{*}{ Pre (\%) } & \multirow[b]{2}{*}{ Post (\%) } & \multicolumn{2}{|c|}{ Relative change } \\
\hline & & & & & $\%$ & p Value & & & & & $\%$ & p Value \\
\hline \multicolumn{13}{|l|}{ Car users } \\
\hline Original model & $0.88(0.83$ to 0.93$)$ & $<0.001$ & $0.18^{*}$ & -0.19 & -0.37 & 0.005 & $0.84(0.79$ to 0.90$)$ & $<0.001$ & $0.36^{*}$ & 0.13 & -0.23 & 0.106 \\
\hline Exposure-adjusted model & 0.61 (0.51 to 0.72 ) & $<0.001$ & 0.01 & -3.60 & -3.61 & $<0.001$ & 0.60 (0.50 to 0.71 ) & $<0.001$ & 0.18 & -3.27 & -3.45 & $<0.001$ \\
\hline Exposure-adjusted model & 0.64 (0.55 to 0.73 ) & $<0.001$ & $-0.33^{*}$ & $-1.54^{*}$ & -1.21 & $<0.001$ & 0.60 (0.51 to 0.72 ) & $<0.001$ & -0.27 & $-1.32^{*}$ & -1.05 & 0.003 \\
\hline \multicolumn{13}{|c|}{ Moped users } \\
\hline Original model & $0.99(0.93$ to 1.04$)$ & 0.607 & $-0.80^{*}$ & -0.12 & 0.68 & $<0.001$ & $1.02(0.95$ to 1.09$)$ & 0.628 & $-0.55^{*}$ & -0.09 & 0.46 & 0.001 \\
\hline Exposure-adjusted model & $0.68(0.59$ to 0.78$)$ & $<0.001$ & $-0.89 *$ & $-3.58^{*}$ & -2.69 & $<0.001$ & $0.71(0.61$ to 0.82$)$ & $<0.001$ & $-0.69^{*}$ & -3.59 & -2.90 & $<0.001$ \\
\hline
\end{tabular}

Post, post-intervention period; Pre, pre-intervention period. Pre-intervention period: 1 January 2000-31 December 2003; post-intervention period: 1 January $2004-31$ December 2006. ${ }^{*} \mathrm{p}<0.05$.

†Time trends in the pre (Pre(\%)) and post (Post(\%)) intervention periods are expressed as the mean percentage increase/decrease in the number of people injured per month, statistically significant trends being indicated with an asterisk $\left({ }^{*}\right)$. Relative change is expressed as the mean percentage increase/decrease in the number of people injured per month in the postintervention period compared with that in the pre-intervention period. 
among motorcycle and moped users, although they are probably due to exposure variations, because the exposure-adjusted models showed significant risk reductions among these road users. In fact, the series for injured motorcycle and moped users is very similar to that for motorcycle and moped registrations. Moreover, similar figures have been observed in Europe: motorcycle fatalities increased 5.3\% in 2006 (compared with 2000), and the proportion of injured moped users decreased substantially during 2000-3 and later stabilised. ${ }^{24}$ Nonetheless, exposure-adjusted results should be interpreted with caution because this denominator-although the best available-does not reflect changes in road users' mobility, but only in the number of available vehicles.

Finally, no effect was observed among pedestrians. This seems reasonable, because road safety interventions were mainly focused on vehicles (eg, speed cameras, alcohol sobriety checkpoints) and on non-urban roads.

\section{Short and long-term effectiveness}

Both short and long-term effectiveness was observed. This reveals that effective measures were implemented at the beginning of the year 2004 (short-term effect). In addition, other interventions implemented throughout the post-intervention period were also effective, progressively reducing the number of people injured (time trend; long-term effect).

\section{Limitations and strengths}

The number of vehicle-kilometres could not be used. However, vehicle fleet and new vehicle registrations were used as proxies for exposure. Also, the increased effort made from the police department to improve the reporting of collisions probably affected the results.

No comparison group was available, as the evaluation was nationwide. Nonetheless, although it may add evidence to the results, it is not compulsory when using time series analysis, as percentage change is only compared among time points in the same series.

Uncontrolled factors could be influencing the results. However, several socioeconomic variables were accounted for as potential confounding factors. Only fuel consumption was statistically associated with the series of people injured and did not modify the results noticeably (data not shown).

The validity of the results are subject to data quality. Misclassification among mopeds and motorcycles has been observed previously in the police database, ${ }^{25}$ and also with injury severity data: one third of seriously injured people are classified as being slightly injured. ${ }^{26}$ The small number of missing values regarding sex $(3.4 \%)$ are not expected to have significantly affected the results. Moreover, this number was approximately $4 \%$ between 2000 and 2004, and dropped to $1.8 \%$ and $1 \%$ in 2005 and 2006, respectively, which goes against our hypothesis.

Among the strengths of the study, the design and the statistical analysis performed allowed us to control for the main confounding factors that usually affect road safety evaluation studies, such as regression to the mean and general trends in the number of crashes. ${ }^{27}$ Although other authors suggest using autoregressive integrated moving average (ARIMA) models. ${ }^{28}$ Poisson regression has been observed to yield similar estimates with a similar goodness of fit of the models. Moreover, their coefficients can be interpreted in terms of relative risks, which provide a straightforward interpretation of the effectiveness of an intervention. ${ }^{29}{ }^{30}$ In addition, the use of two different RR (one for mean change and another for time trend), compared with the use of only one RR, allows us to distinguish between

\section{What is already known on this subject}

A strong political will is required to reduce road traffic injuries.

- However, to date, the overall effect of the actions implemented following the prioritisation of road safety has not been assessed.

\section{What this study adds}

The present study suggests that the inclusion of road safety on the political agenda (and the subsequent implementation of several road safety interventions) reduces the number of people injured in road traffic collisions.

- In Spain, 24629 people injured and 1642 deaths due to road traffic collisions were prevented 3 years after road safety prioritisation.

the short and long-term effects. Also, the long pre and postintervention periods available provide stability to the analysis. In addition, a large sample size was available, allowing for subgroup analyses. Finally, the hospital discharge registry was also analysed to improve the validity of the results among seriously injured people, ${ }^{31}$ yielding similar results to those obtained with the police database, adding consistency to the results; the risk of being admitted to hospital due to traffic collisions in the post-intervention period was close to one regarding the mean number for both men (RR 0.98; 95\% CI 0.93 to 1.04 ) and women (RR $0.98 ; 95 \%$ CI 0.91 to 1.05 ), and a statistically significant reduction was observed regarding the time trend $(0.41 \%$ and $0.82 \%$ monthly reduction, respectively).

\section{CONCLUSIONS}

Overall, road safety interventions implemented following the inclusion of road safety on the political agenda reduced the number of traffic-injured people, thus suggesting the effectiveness of road safety prioritisation. Further studies should assess the differences in the effectiveness between road types, geographical area and type of measures implemented, which would help resolve which combinations of road safety strategies are the most effective in reducing traffic injuries.

Funding This work was supported by the Agencia Española de Tecnologías Sanitarias (Plan Nacional de Investigación Científica, Desarrollo e Innovación Tecnológica (I+D +I) e Instituto de Salud Carlos III-Subdirección General de Evaluación y Fomento de la Investigación) (PI07/90157). The funding source has not had any involvement in the study design, in the collection, analysis and interpretation of data, in the writing of the report and in the decision to submit the paper for publication.

\section{Competing interests None.}

Ethics approval The present study was approved by the ethics committee Comitè Etic d'Investigació Clínica - IMAS, of the Institut Municipal d'Assistència Sanitària (IMAS).

Contributors All authors fulfilled the criteria of authorship. AMN, KP, ES-R, MM-DO AT and CB designed the study. AMN performed the statistical analyses. All of the authors contributed in the interpretation and the discussion of the results. AMN wrote the first draft of the paper. All of the authors critically revised the manuscript and approved the final version of the manuscript.

Provenance and peer review Not commissioned; externally peer reviewed.

\section{REFERENCES}

1. Peden M, Scurfield R, Sleet D, et al, eds. World report on road traffic injury prevention. Geneva: World Health Organization, 2004. 
2. Lopez AD, Mathers CD, Ezzati M, et al. Global and regional burden of disease and risk factors, 2001: systematic analysis of population health data. Lancet 2006;367:1747-57.

3. Peiró R, Seguí-Gómez M, Pérez K, et al. Lesiones por tráfico, de ocio y domésticas y laborales. Descripción de la situación en España. Informe SESPAS 2006. Gac Sanit 2006;20(Suppl 1):32-40.

4. Cubí $\mathbf{P}$, Herrero C. Evaluación de riesgos y del impacto de los accidentes de tráfico sobre la salud de la población española, 1964-2004 [dissertation]. Bilbao: Fundación BBVA, 2008.

5. Llàcer A, Fernández-Cuenca R. Martínez de Aragon MV. Mortalidad en España en 1998. Evolución en la década. (I) Mortalidad General, principales causas de muerte y años potenciales de vida perdidos. Boletín Epidemiológico Semanal 2001;9:241-4

6. European Commission. White Paper. European transport policy for 2010: time to decide. Luxembourg: Office for Official Publications of the European Communities, 2001.

7. European Commission. Saving 20000 lives on our roads. A shared responsibility. European Road Safety Action Programme. Halving the number of road accident victims in the European Union by 2010: a shared responsibility. Luxembourg: Office for Official Publications of the European Communities, 2003.

8. Joint OECD/ECMT Transport Research Centre. Road Safety. Achieving ambitious road safety targets. Paris: Organisation for Economic Co-operation and Development, 2006.

9. ECORYS Transport and SWOV. Impact assessment road safety action programme. Assessment for mid term review. Rotterdam: ECORYS Transport, 2005.

10. European Transport Safety Council. A methodological approach to national road safety policies. Brussels: European Transport Safety Council, 2006.

11. Dirección General de Tráfico. Ministerio de Interior. Plan Estratégico de Seguridad Vial 2005-2008. Medidas Especiales de Seguridad Vial 2004-2005. Madrid Dirección General de Tráfico; 2005

12. Dirección General de Tráfico. Ministerio de Interior. Plan Estratégico de Seguridad Vial 2005-2008. Plan de Acciones Estratégicas Claves 2005-2008. Madrid: Dirección General de Tráfico, 2006.

13. Villalbí JR, Pérez C. Evaluation of regulatory policies: the prevention of traffic accidents in Spain. Gac Sanit 2006;20(Suppl 1):79-87.

14. Dirección General de Tráfico. Ministerio de interior. Memoria de actuaciones de seguridad vial 2005. Madrid: Dirección General de Tráfico, 2006.

15. Dirección General de Tráfico. Ministerio de interior. Memoria de actuaciones de seguridad vial 2006. Madrid: Dirección General de Tráfico, 2007.
16. Rodríguez JI. Del viejo código al permiso por puntos. Tráfico y seguridad vial 2009; 197:42-3.

17. Dirección General de Tráfico. http://www.dgt.es/portal/es/seguridad vial/ estadistica/matriculaciones definitivas/ (accessed Sep 2008).

18. Yannis G, Antoniou C, Papadimitriou E. Road casualties and enforcement: distributional assumptions of serially correlated count data. Traffic Inj Prev 2007; 8:300-8.

19. Stolwijk AM, Straatman H, Zielhuis GA. Studying seasonality by using sine and cosine functions in regression analysis. J Epidemiol Community Health 1999:53:235-8.

20. Langbein LI, Felbinger CL. The quasi experiment. In: Langbein LI, ed. Public program evaluation: a statistical guide. New York: ME Sharpe, 2006:106-33.

21. StataCorp. Stata Statistical Software: release 10 [computer program]. College Station, TX: StataCorp LP, 2005.

22. Jonah BA. Sensation seeking and risky driving: a review and synthesis of the literature. Accid Anal Prev 1997:29:651-65.

23. Laapotti S, Keskinen E. Differences in fatal loss-of-control accidents between young male and female drivers. Accid Anal Prev 1998;30:435-42.

24. European Road Safety Observatory. Annual Statistical Reports 2007. Brussels: European Road Safety Observatory, 2008.

25. Pérez K, Marí-Dell'Olmo M, Borrell C. Road injuries and relaxed licensing requirements for driving light motorcycles in Spain: a time-series analysis. Bull World Health Organ 2009;87:497-504.

26. Pérez K, Cirera E, Borrell C, et al; on behalf of the work group of the Spanish Society of Epidemiology on the Measuring of the Impact on health of road traffic accidents in Spain. Motor vehicle crash fatalities at 30 days in Spain. Gac Sanit 2006:20:108-15.

27. Hauer E. Observational before-after studies in road safety: estimating the effect of highway and traffic engineering measures on road safety. Oxford, England: Pergamon Press, 2005.

28. Dupont $\mathbf{E}$, Martensen $\mathrm{H}$, eds. Multilevel modeling and time series analysis in traffic research-methodology. Deliverable D7.4 of the EU FP6 project. Brussels: European Road Safety Observatory, 2007

29. Tobias A, Díaz J, Saez M, et al. Use of poisson regression and box-jenkins models to evaluate the short-term effects of environmental noise levels on daily emergency admissions in Madrid, Spain. Eur J Epidemiol 2001:17:765-71.

30. Kuhn L, Davidson LL, Durkin MS. Use of Poisson regression and time series analysis for detecting changes over time in rates of child injury following a prevention program. Am J Epidemiol 1994;140:943-55.

31. Lyons RA, Ward $\mathrm{H}$, Brunt $\mathrm{H}$, et al. Using multiple datasets to understand trends in serious road traffic casualties. Accid Anal Prev 2008:40:1406-10. 